\title{
Diferencias en la lectura conjunta \\ adulto-niño y su relación con el estilo \\ cognitivo del adulto*
}

//Differences in adult-child shared reading

Revista Colombiana

Investigaciones

de Educación, N. 64

Primer semestre de 2013,

Bogotá, Colombia. and its relationship with adult cognitive style

//Diferençias na leitura conjunta adulto-

criança e sua relação com o estilo cognitivo

do adulto

Nicolás Arias Velandia**
Recibido: 21/11/2012

Evaluado: 31/01/2013 - 15/02/2013

\begin{abstract}
Artículo derivado del trabajo de grado presentado por el autor para optar al título de Magíster en Educación, Grupo Estilos Cognitivos, Universidad Pedagógica Nacional, Bogotá, Colombia.

Magíster en Educación. Coordinador del Observatorio de Educación, Politécnico Gran Colombiano Institución Universitaria. Correo electrónico: nariasv@gmail.com
\end{abstract}

\section{Resumen}

Esta investigación caracterizó diferencias en la lectura conjunta de doce díadas adulto-niño y su relación con el estilo cognitivo de los adultos de dichas díadas. Se realizaron seis sesiones de lectura conjunta libre (sin dirección del investigador) de hora y media, registrando acciones del adulto y del niño en una guía de observación al inicio y al final de las mismas. Se utilizaron las pruebas de figuras enmascaradas (embedded figures test, EFT) y del marco y la varilla (rod and frame test, RFT) para calcular el estilo cognitivo del adulto. Los resultados mostraron dos tendencias en la interacción de las díadas: oscilación entre la focalización en el libro como objeto y la focalización en la interacción misma, y oscilación entre atención a la narración y atención a los aspectos gráficos del libro. Solo la primera tendencia tuvo una leve correlación con el estilo cognitivo del adulto. Se discuten los alcances y posibilidades de estas dimensiones como estilos en la interacción adulto-niño preescolar.

\section{Abstract}

This study described differences in shared reading of 12 adult-child dyads and its relationship with the cognitive style of the adults in these dyads. Six sessions of free shared reading (non-directed and non-instructed by the researcher) of one and a half hour were performed, registering adult and child actions in an observation guide format at the beginning and at the end of the sessions. Adult cognitive style was obtained with the embedded figures test (EFT) and the rod and frame test (RFT). The results showed two tendencies in the dyads' interactions: oscillation between focus on the book as an object and focus on interaction itself, and oscillation between attention to narrated story and attention to graphic aspects in the book. Only the first tendency had a low correlation with adult cognitive style. The scopes and possibilities of these dimensions as styles in the adult-preschooler interaction are discussed.

\section{Resumo}

Esta pesquisa caracterizou as diferenças na leitura conjunta de doze duplas adultocriança e sua relação com o estilo cognitivo dos adultos de tais duplas. Realizaram-se seis sessões de leitura conjunta livre (sem orientação do pesquisador) de hora e meia, registrando ações do adulto e da criança numa guia de observação ao começo e ao final das mesmas. Utilizaram-se os testes: (embedded Figures Test-EFT) e (Rod and Frame Test-RFT) para avaliar o estilo cognitivo do adulto. Os resultados mostraram duas tendências na interação das duplas: oscilação entre a focalização no livro como objeto e a focalização na interação mesma, e oscilação entre atenção à narração e atenção aos aspectos gráficos do livro. Só a primeira tendência teve uma leve correlação com o estilo cognitivo do adulto. Discutem-se os alcances e possibilidades destas dimensões como estilos na interação adulto-criança pré-escolar.

\section{Palabras Clave}

Lectura conjunta, interacción adulto-niño, estilos cognitivos, independencia-sensibilidad al medio

\section{Keywords}

Shared reading, adult-child interaction, cognitive styles, field independence-sensitivity.

\section{Palavras chave}

Leitura conjunta, interação adulto-criança, estilos cognitivos, independência-sensibilidade ao entorno. 


\section{Introducción}

La lectura conjunta es una interacción entre un niño y un adulto en la cual ambos observan y leen libros conjuntamente y conversan sobre ellos (Bus, 2002). En dicha interacción ambos aportan, pero es el adulto quien le da dirección y propósito, convirtiéndola en una actividad que promueve conocimientos iniciales sobre la lectura (Flórez, Restrepo y Schwanenflugel, 2007). Por tanto, es posible que las diferencias en la acción de los adultos durante la lectura conjunta se relacionen con sus características individuales y con el tipo de interacción que generan con el niño (Bus, 2002; Flórez, Castro y Arias, 2009).

Este estudio se interesa por las diferencias en la lectura conjunta llevada a cabo por díadas de padres e hijos y la manera en la cual dichas diferencias se relacionan con el estilo cognitivo del adulto en la dimensión independencia-sensibilidad al medio.

La lectura conjunta promueve los conocimientos alfabéticos iniciales. Su despliegue o curso depende del conocimiento previo que tienen sus participantes sobre dicha actividad (Bus, 2002; Bus y Sulzby, 1996), de las ideas y prácticas de los adultos en el hogar sobre lectura (Flórez et al., 2006a; Wade y Moore, 2000; Collins et al., 2005), del ideal de formación que tienen tales adultos con respecto al niño (Lautrey, 1985; Páramo y Tinajero, 1990, 1992; Páramo et al., 1991), del nivel educativo de los padres (Flórez et al., 2006b), del posible valor dado a la lectura en el oficio de los padres o adultos cercanos (e.g., estar más o menos cerca de ocupaciones intelectuales como el magisterio, la investigación científica, el derecho, la revisión de textos, entre otros) y de características personales o individuales del adulto que le lee (Bus, 2002; cf. Hederich y Camargo, 1999; cf., Hederich, 2007). La relación que se crea y se mantiene entre los participantes en esta situación generalmente determina la relación del niño con el adulto y con las actividades de lectura (Bus, 2002).

Varias investigaciones han encontrado que la lectura conjunta favorece en el niño el crecimiento de su vocabulario, su conocimiento de la forma del lenguaje escrito, su relación de apego con el adulto con quien la realiza, su uso del lenguaje oral en torno a un tópico, su razonamiento sobre eventos y situaciones, su conocimiento de formas de expresión lingüística no familiares en el entorno cercano y su interés por la lectura y por activida- 
des de aprendizaje en interacción con los adultos (Wells, 1981, 1985; Wade y Moore, 2000; Collins et al., 2005; Bus, 2002; Flórez et al., 2006a).

La interacción que define la lectura conjunta suele tener tres momentos: el comienzo, el curso o desarrollo de la misma y la finalización. En el comienzo el niño o el adulto toman un nuevo libro y en la finalización el niño o el adulto deciden finalizar la actividad y dejan el libro. También se considera como parte analizable de este fenómeno el grado de involucramiento que se logra del niño en la actividad (Flórez et al., 2006a), las acciones y las reacciones del niño y del adulto en la actividad y estados emocionales del pequeño durante la misma (Perinat, 1999) y la focalización de dichas acciones en la interacción o en el contenido del material explorado (Bus, 2002). En la tabla 1 se muestran dichos aspectos en relación con los niveles de análisis que se acaban de enunciar (anexo 1, instanciación específica en acciones a observar y registrar).

Tabla 1. Momentos en la lectura conjunta y aspectos observados en cada uno de ellos.

\begin{tabular}{l|l}
\hline Momento en la lectura conjunta & \multicolumn{1}{c}{ Aspectos observados } \\
\hline Comienzo de la actividad & $\begin{array}{l}\text { 1. Muestras de algún estado o emoción particular en el niño al } \\
\text { comenzar la actividad. } \\
\text { 2. Decisión del adulto de tomar libro o dejar que el niño lo tome. }\end{array}$ \\
\hline $\begin{array}{l}\text { 1. Forma en que el adulto desarrolla la lectura con el niño. } \\
\text { 2. Modificaciones de voz del adulto para leer con el niño. } \\
\text { 3. El adulto muestra todo o solo parte del contenido del libro al niño. } \\
\text { 4. Tipo de sensación mostrada por el niño mientras se desarrolla la } \\
\text { actividad. } \\
\text { 5. Tipo de actividad predominante en el niño. } \\
\text { 6. Aspecto sobre el cual habla el niño en la interacción. } \\
\text { 7. Acción específica que predomina en el niño durante la lectura } \\
\text { conjunta. }\end{array}$ \\
\hline Finalización de la actividad & $\begin{array}{l}\text { 1. Necesidad del niño de continuar la actividad a pesar de marcar su } \\
\text { finalización. } \\
\text { 2. Reacción del niño cuando el adulto da por finalizada la actividad. } \\
\text { 3. Involucramiento de otro adulto en la lectura. }\end{array}$ \\
\hline $\begin{array}{l}\text { Observaciones posteriores a la } \\
\text { actividad }\end{array}$ & $\begin{array}{l}\text { 1. Tipo de expresión del niño durante toda la actividad. } \\
\text { 2. Acción que suele predominar en el adulto durante la lectura } \\
\text { conjunta. }\end{array}$ \\
\hline niño en la actividad realizada & 1. Juicio del observador sobre el grado de involucramiento del niño. \\
\hline
\end{tabular}

También se mencionó la relación entre diferencias individuales en el curso que suele tomar la lectura conjunta. Con frecuencia se han mostrado diferencias de nivel entre las díadas que realizan lectura conjunta (por ejemplo, mayor o menor nivel de involucramiento y desarroIlo de la lectura conjunta) (Wells, 1981, 1985; Wade y Moore, 2000; 
Collins et al., 2005; Flórez et al., 2006a) ${ }^{1}$, pero también podrían suponerse diferencias de estilo ${ }^{2}$ en los cursos de interacción en díadas adulto-niño si se tiene en cuenta que su despliegue puede relacionarse con características individuales del adulto (Bus, 2002) como su tendencia de estilo cognitivo independiente del medio o sensible al medio (cf. Saracho, 2000, 2003 ) .

Los estudios sobre el estilo cognitivo de los adultos en la dimensión independencia-sensibilidad al medio, muestran que en cada uno de los polos de esta dimensión hay patrones consistentes de actuación de los adultos en la interacción que siguen con los niños pequeños y en actividades con fines de aprendizaje que realizan con ellos (Saracho, 2000, 2003; cf. Páramo y Tinajero, 1990, 1992; Páramo et al., 1991)4. Los adultos de estilo cognitivo sensible al medio tienden a privilegiar o valorar la interacción en sí misma y los de estilo independiente del medio el cumplimiento de metas más allá de la interacción. En ese sentido, los profesores de educación inicial sensibles al medio realizan más actividades con los niños que estos disfruten y a buscar el contacto uno a uno con cada uno de ellos, y los independientes del medio realizan con ellos actividades en las que tengan que resolver tareas o situaciones por sí mismos, y a dirigirse a todos los niños en general (Saracho, 2000, 2003).

De acuerdo con las tendencias de focalización de la atención del adulto en la lectura conjunta identificadas por Bus (2002), los adultos sensibles al medio preferirían las actividades de mantenimiento de la interacción en la lectura conjunta, mientras que los independientes del medio atenderían más al contenido. Dichas tendencias también pueden tener influencia de las ideas,

1 Las diferencias de nivel se relacionan con conceptos de valoración del desempeño con respecto a un criterio general, como inteligencia (por ejemplo, valoración de la eficacia de la persona para resolver tareas) o estrategia (actos planeados dirigidos a una meta particular que desarrollan cambios en los modos de acción en función de los cambios en las circunstancias).

2 Las diferencias de estilo en este caso hacen referencia a la presencia de diferentes modalidades o tipos de interacción adulto-niño en las cuales ninguna es mejor o peor que otra.

3 En este campo de estudio, estilo se define como una forma de acción que tiene dos o más tendencias de manifestación entre las personas, que permanece a través de distintos campos o dominios, que permanecen a lo largo del tiempo y de distintas situaciones que la persona enfrenta y ninguna de sus maneras de manifestarse es mejor o peor que otra (solamente es diferente). Es un concepto semejante a los de personalidad o características individuales (Hederich, 2007)

4 El estilo cognitivo se define como un conjunto consistente de regularidades en la forma de actuar y de procesar información en una persona. Es la dimensión de estilo cognitivo más estudiada, con más evidencias empíricas favorables, y la que ha planteado un modelo integrador de diferentes dimensiones de la acción (solución a tareas, bases neuropsicológicas, interacción social; para una descripción más detallada, cf. Hederich, 2007). 
creencias y prácticas de los adultos en el hogar. Por este motivo, en esta investigación nos preguntamos: ¿qué relación existe entre el curso y dinámica de la lectura conjunta y el estilo cognitivo del adulto en la díada adulto-niño?

\section{Método}

Este estudio se divide en dos bloques: descriptivo y correlacional. En el bloque descriptivo se realiza una caracterización de la frecuencia de aparición de las acciones en la lectura conjunta inicial, sus asociaciones más importantes y su mayor o menor tendencia a la estabilidad con el paso del tiempo. Busca ubicar acciones en la lectura conjunta con mayor variación entre las díadas participantes para observar luego si se agrupan de acuerdo con una tendencia diferencial.
En el bloque correlacional se busca establecer la manera en que las acciones en la lectura conjunta se asocian con el estilo cognitivo en la dimensión independencia-sensibilidad al medio.

\section{Diseño de investigación}

En este trabajo se observaron y se registraron las acciones de las díadas adulto-niño participantes durante la primera y la quinta de una serie de sesiones de lectura conjunta realizadas una vez por semana en un jardín infantil del campus de una universidad pública en Bogotá D.C., Colombia. En una sesión previa a las destinadas exclusivamente a la observación, los padres respondieron las pruebas de estilo cognitivo en la dimensión independencia-sensibilidad al medio. El diseño de esta investigación se ilustra en la figura 1.

Figura 1. Diseño de la investigación.

\begin{tabular}{|c|c|c|c|c|}
\hline Semana 1 & Semana 2 & Semana 3 & Semana 4 & Semana 5 \\
\hline $\begin{array}{l}\text { Observación de } \\
\text { acciones en } \\
\text { lectura conjunta } 1\end{array}$ & \multicolumn{3}{|c|}{ Desarrollo de sesiones de lectura conjunta } & $\begin{array}{c}\text { Observación de } \\
\text { acciones en } \\
\text { lectura conjunta } 2\end{array}$ \\
\hline \multicolumn{5}{|c|}{ Estilo cognitivo del adulto, dimensión independencia-sensibilidad al medio. } \\
\hline \multicolumn{5}{|c|}{ Costumbres y creencias del adulto sobre la lectura. } \\
\hline \multicolumn{5}{|c|}{ Creencias, condiciones y acciones del adulto sobre el aprendizaje de los niños } \\
\hline
\end{tabular}

Este diseño exige la observación de las mismas díadas de adultos y niños al inicio y al final de las sesiones. Esto se realiza con el fin de comparar la estabilidad de las acciones en la lectura conjunta a través del tiempo, su pertenencia a diferentes aspectos de la lectura conjunta, y las tendencias diferenciales en su curso $^{5}$ (que se presentan analizados

5 En la segunda observación, realizada hacia el fina del taller, sólo participaron seis díadas adulto-niño de las doce observadas en los otros puntos, por diversos inconvenientes con horarios y actividades laborales de los padres en la finalización del año académico en la universidad. Por este motivo, las comparaciones de la observación entre dos momentos solamente se realizaron con esas 6 díadas. 
en los resultados del bloque descriptivo). El registro de las acciones en la lectura conjunta se correlacionó con las otras variables mencionadas en el párrafo anterior cuyos datos se obtienen mediante pruebas y cuestionarios (que se presentan analizados en los resultados del bloque correlacional). Adicionalmente se obtuvieron resultados de otras variables potencialmente asociadas con las interacciones en las díadas: costumbres y creencias del adulto sobre la lectura, y creencias, condiciones y acciones del adulto sobre el aprendizaje de los niños.

\section{Participantes}

Participaron doce díadas de adultos cuidadores y niños pertenecientes a un jardín infantil de un campus en una universidad pública en Bogotá D. C., Colombia. Los adultos de estas díadas atendieron la invitación a una convocatoria para participar en un espacio de cinco sesiones para leer con sus hijos, hecha en una reunión de padres de familia convocada por la directora del jardín infantil. Adicionalmente, también se entregó a los adultos una carta que explicaba el objetivo de la actividad a realizar. Al comenzar las sesiones, a los adultos se les pidió consentimiento para participar en la investigación con sus hijos que asisten al jardín infantil.

En las díadas hubo doce adultos en total (ocho padres y cuatro madres) y doce niños (seis varones y seis niñas). Era usual que el niño tuviese un hermano (en cuatro casos era un hermano mayor, en cinco un hermano menor y en tres casos no tenía hermanos). Ocho niños contaban con edades entre 3 y 4 años, y cuarto de ellos tenían de 4 a 5 años.

Cinco díadas reportaron devengar ingresos familiares entre dos y tres salarios mínimos. Dos reportaron entre tres y cuatro salarios mínimos, y otras dos, cuatro salarios mínimos o más. Tres díadas no reportaron ingresos familiares. Los padres de los niños de las díadas registraron edades entre 21 y 40 años, y las madres entre 21 y 30 años.

\section{Variables, instrumentos e indicadores}

En la siguiente tabla se detallan las variables y los instrumentos o indicadores de medida utilizados. Los detalles de la composición de las variables y de los instrumentos se pueden consultar en el anexo 1. 
Tabla 2. Instrumentos utilizados.

\begin{tabular}{l|l}
\multicolumn{1}{c|}{ Variable } & Instrumento o mecanismo de recolección de información \\
\hline Acciones en la lectura conjunta. & $\begin{array}{l}\text { Guía de observación de la actividad de lectura (Flórez et } \\
\text { al., 2006b, en versión modificada para este estudio). }\end{array}$ \\
\hline $\begin{array}{l}\text { Estilo cognitivo en la dimensión } \\
\text { independencia-sensibilidad al medio de } \\
\text { los adultos. }\end{array}$ & $\begin{array}{l}\text { Prueba de figuras enmascaradas (EFT, embedded figures } \\
\text { test, versión Sawa-Gottschaldt). }\end{array}$ \\
\cline { 2 - 3 } $\begin{array}{l}\text { Costumbres y creencias del adulto } \\
\text { sobre la lectura }\end{array}$ & $\begin{array}{l}\text { Prueba del marco y la varilla (RFT, rod and frame test, } \\
\text { versiótil). }\end{array}$ \\
\hline $\begin{array}{l}\text { Creencias, condiciones y acciones del de costumbres de lectura (Flórez et al., 2006b). } \\
\text { adulto sobre el aprendizaje de los niños }\end{array}$ & Cuestionario del ambiente Familiar (Lautrey, 1985). \\
\hline
\end{tabular}

La Guía de observación de la actividad de lectura (basada en Flórez et al., 2006b) contiene 36 ítems (que corresponden a cada una de las variables indagadas en la lectura conjunta inicial) en los cuales un observador externo (ciego a los objetivos del estudio y a los detalles de la investigación) identifica si el aspecto mencionado en cada ítem se presenta. Cuando se presenta, el observador marca una equis en frente de dicho aspecto (anexo 1). Cuando no se presenta, deja sin marcar dicho aspecto. Posteriormente, en la base de datos, los aspectos observados de la lectura conjunta son calificados con 2 cuando se presentan, y con 1 cuando no se presentan. El observador registra una unidad de interacción en cada rejilla, donde cada unidad de interacción se considera una interacción adulto-niño leyendo u observando un libro particular. Cada interacción comienza cuando se toma un libro particular y finaliza cuando dicho libro se deja para terminar la actividad.
Cada interacción se registra teniendo en cuenta el comienzo, el desarrollo y la finalización de la actividad. Se registra además un apartado de juicios del observador sobre la interacción. El instrumento enuncia varias acciones opuestas sobre una misma tendencia para que el observador estime cuál tendencia predominó más en la actividad observada (por ejemplo, "el adulto escoge un libro para el niño" $y$ "el adulto permite o invita al niño a que él mismo escoja el libro") ${ }^{6}$. Estas observaciones fueron realizadas a cada pareja por dos analizadores,

6 Se optó por este procedimiento de registro porque: 1) permite a observadores tener una impresión global sobre la interacción sin diferirla en el tiempo y sin interrumpir la actividad del observador para registrar información; 2) permite a observadores escoger una sola tendencia que predomine más entre dos polos o entre un conjunto de opciones sobre un mismo aspecto, y 3) muestra una confiabilidad entre observadores mucho más alta que otras usadas en estudios anteriores (Flórez et al., 2006b). Esta decisión sobre el instrumento tomó más fuerza porque las dos observadoras realizaron su labor sin conocer de qué trataba la investigación ni cuáles eran las hipótesis del trabajo. 
estudiantes de último semestre de fonoaudiología; ambas observaban simultáneamente a cada díada en la lectura conjunta y registraban su observación desde lados contrarios del salón donde estaban las díadas. Ambas observadoras desconocían el estudio, desconocían el problema de investigación y también otros datos de caracterización de las díadas, como el nivel socioeconómico de la familia, el nivel educativo de los adultos. También desconocían el estilo cognitivo de estos (que fue examinado por el investigador en una sesión aparte). Con los registros tomados por ambas observadoras en la guía de observación se realizaron cálculos de confiabilidad entre observadores (kappa $=90 \%$ ).

La prueba de figuras enmascaradas (EFT) y la prueba del marco y la varilla (RFT) se utilizan para determinar el estilo cognitivo del adulto participante en la díada de acuerdo con el procedimiento expuesto en Hederich (2007). Estas fueron aplicadas por uno de los investigadores sin conocer todavía los datos de otras observaciones o cuestionarios aplicados para caracterizar a las díadas.

En los dos primeros aspectos se les pide a los padres que seleccionen de tres opciones dadas, la que mejor corresponde a su vida en el hogar, y en los dos últimos escoger, por orden de prioridad, tres aspectos de diez posibles. Con esto se busca observar que ideales se valoran más sobre el tipo de aprendizaje que se imparte al niño en el hogar (anexo1); adicionalmente, con este instrumento se recolectan datos de ocupación de los padres y del nivel educativo máximo que han alcanzado.

\section{Procedimiento de recolección de datos}

Las actividades de esta investigación se desarrollaron en seis sesiones de una hora y media por semana. En una primera sesión se realizó la caracterización del estilo cognitivo de los cuidadores, se aplicaron los dos cuestionarios sobre ideas y prácticas sobre lectura de adultos y niños en el hogar, sobre el ideal de formación del niño que tienen los adultos en el hogar, y se resolvieron dudas de los padres a acerca de su participación en esta experiencia. En las otras cinco sesiones se desarrollaron actividades de lectura conjunta de adultos y niños, con eventuales charlas de los padres con el investigador sobre la promoción de conocimiento con la lectura conjunta para fortalecer el vínculo interpersonal entre el niño y el adulto, y sobre el conocimiento de los libros disponibles para leer con los niños. En la segunda 
y la sexta sesión se llevaron a cabo observaciones de la lectura conjunta inicial en la Guía de observación de la lectura conjunta inicial. A las observadoras no se les permitió conocer objetivos del estudio, ni ninguna información concerniente a otras aspectos de esta y se les instruyó para que registraran en la rejilla de la guía de observación "la tendencia que más predomine en la interacción entre el adulto y el niño luego de observarlos desde que toman un determinado libro hasta que lo dejan" (ver "Instrumentos").

\section{Estrategias de análisis}

La información se analizó de acuerdo con los dos bloques que componen el estudio. Para el análisis en el bloque descriptivo se utilizaron:

- Datos descriptivos de la frecuencia de acciones en la lectura conjunta.

- Prueba no paramétrica $W$ de Wilcoxon para muestras pareadas en las seis díadas observadas en la primera sesión y en la última sesión para ver la tendencia a la estabilidad temporal en las acciones de la lectura conjunta.

Para el bloque correlacional se usaron:

- Correlaciones de Spearman entre estilo cognitivo de los padres y acciones en la lectura conjunta.
- Análisis de correspondencias múltiples, con el estilo cognitivo del adulto y con las variables de relación de la familia con la lectura y con ideales educativos con el niño como variables descriptivas, para indagar las tendencias de agrupación en las acciones durante la lectura conjunta y la relación de estas tendencias de agrupación con el estilo cognitivo del adulto.

- Análisis de correspondencias múltiples sobre acciones en la lectura conjunta, respuestas a los cuestionarios sobre ideas y prácticas de lectura de adultos y niños en el hogar, e ideales de formación de los niños que tienen los adultos en el hogar.

\section{Resultados y \\ discusiones por bloques}

\section{Bloque descriptivo}

Se presentan 6 acciones en todas o casi todas las díadas (en diez o más díadas): el niño presenta buena disposición en su estado de salud y de ánimo (doce díadas), el niño participa activamente en la actividad (once díadas), el adulto lee con el niño conversando activamente con este (once díadas), el adulto prefiere hablar con el niño sobre los dibujos (diez casos), y el adulto lee o muestra todo el contenido del libro al niño (diez casos) (figura 2). 
Se presentan dieciocho acciones con muy baja frecuencia en las díadas (con una frecuencia de aparición en dos o menos díadas) (figura 2), razón por la cual se excluyeron de este análisis. Entre ellas están: el adulto prefiere guiar la actividad dejando que el niño sostenga el libro, el niño toma otro libro y le pide al adulto que se lo lea al finalizar la actividad, el niño todo el tiempo explora diferentes libros, el niño habla con el adulto de la parte escrita del libro, el niño intenta reproducir solo aquello que el adulto le ha leído, el niño toma el libro en sus manos, entre otras.

Figura 2. Acciones observadas durante la lectura conjunta.

\section{Acciones observadas durante la lectura conjunta}

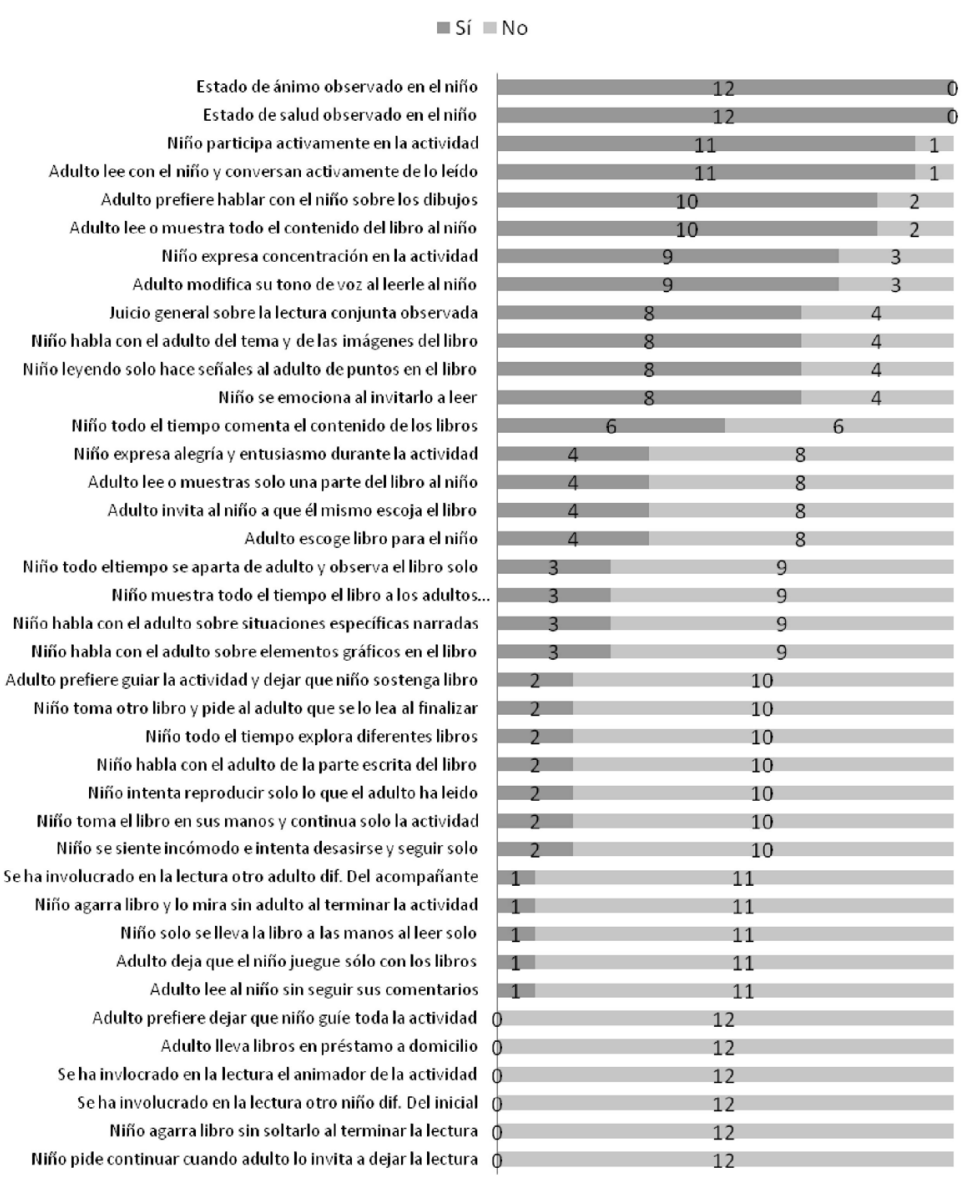


De otra parte, quince acciones que se presentaron solo en algunas díadas (con una frecuencia que va de su presencia de tres a nueve díadas). Entre estas, seis aparecen con más frecuencia (entre nueve y ocho díadas las presentan): el niño expresa concentración en la actividad, el adulto modifica su tono de voz al leer con el niño, el niño permanece atento todo el tiempo durante la realización de la actividad, el niño habla con el adulto del tema y de las imágenes del libro, el niño solamente hace señales al adulto en puntos específicos del libro durante la lectura y el niño se emociona al invitarlo a leer.

También hay una acción, de las quince anteriores, que se presenta con una frecuencia intermedia: el niño todo el tiempo realiza comentarios sobre el contenido de los libros. Así mismo, ocho acciones, de las quince que se presentan en algunas díadas, que se presentan con una frecuencia menor: el niño expresa alegría y entusiasmo durante la actividad, el adulto lee o muestra solo una parte del libro al niño, el adulto invita al niño a que él mismo escoja el libro, el adulto escoge el mismo el libro para el niño, el niño se aparta del adulto y observa el libro sólo todo el tiempo, el niño muestra el libro a los adultos acompañantes todo el tiempo, el niño habla con el adulto sobre situaciones específicas narradas y el niño habla con el adulto sobre elementos gráficos en el libro.
De acuerdo con el cálculo estadístico no paramétrico de comparación de muestras pareadas $W$ de Wilcoxon, no se presentan diferencias significativas en la frecuencia de aparición de las acciones en el primero y el segundo momento de observación en seis de las doce díadas participantes (solo $Z=-2,333$; $p=0,020$ para el grado de involucramientos del niño en la actividad). Las siguientes nueve, de las quince acciones analizadas, tienen una tendencia más clara a aparecer en las díadas tanto en el primero, como el segundo momento de observación:

1. El niño habla con el adulto sobre situaciones específicas narradas.

2. El adulto invita al niño a que él mismo escoja el libro.

3. El adulto lee o muestra solo una parte del libro al niño.

4. El adulto escoge el libro para el niño.

5. El adulto modifica su tono de voz al leerle al niño.

6. El niño leyendo solamente hace señales al adulto de puntos en el libro.

7. El niño habla con el adulto sobre elementos gráficos en el libro.

8. El niño todo el tiempo se aparta del adulto y observa el libro solo.

9. El niño expresa concentración en la actividad. 


\section{Discusiones del bloque descriptivo}

Los resultados muestran que en las acciones en la lectura conjunta hay aspectos universales (que se presentan en casi todas las díadas), otros de aparición variable (que se presentan solo en algunas díadas) y otros de aparición poco frecuente (que se presentan en muy pocas díadas). Los aspectos universales parecen estar relacionados con el mantenimiento de la interacción en la lectura conjunta y en los aspectos que se consideran relevantes en lo leído (Wade y Moore, 2000; Bus, 2002; Watson, 2002; Collins et al., 2005; Flórez et al., 2006b; Flórez et al., 2007).

Los aspectos de aparición variable parecen estar más relacionados con actividades en las cuales se reporta una frecuencia más variable de aparición en la lectura conjunta a través de su realización en diferentes interacciones (Sénéchal et al., 1996; Bus, 2002). En este estudio se trató de especificar de la manera más precisa posible este tipo de acciones, pero en otros estudios similares no es frecuente hallar estas precisiones (Bus y Sulzby, 1996; Flórez et al., 2007; Leseman y De Jong, 1998; Cuetos, 2002; Dickinson et al., 2003; Evans et al., 2000; Storch y Whitehurst, 2002; Van Kleeck, 2003), por lo cual se hace necesario trabajar sobre la validación de estos elementos observados como aspectos o acciones propias de la lectura conjunta.

Es importante destacar que algunas acciones dirigidas a conversar y preguntar sobre el contenido específico de los textos, situaciones que se observan en la interacción en algunas ocasiones, se encuentran entre los aspectos universales y en otras entre los de aparición variable (Cuervo, Flórez y Acero, 2004). Es necesario precisar la relación entre estas acciones y otros indicadores de competencia en los acercamientos iniciales a la lectura, ya que es frecuente que este tipo de acciones se encuentren más relacionadas con díadas adulto - niño que practican con frecuencia la lectura conjunta (Wells, 1981, 1986; Wade y Moore, 2000; Cuervo et al., 2004; Collins et al., 2005; Flórez et al., 2006b).

Los resultados dan cuenta, hasta este punto, de características de estilo en algunas acciones en la lectura conjunta, porque muestran patrones diferenciales y porque estos se dan en varios de los aspectos considerados en la lectura conjunta (Hederich, 2007). Otro indicador para ser considerado dentro de las diferencias de estilo es la estabilidad o permanencia de sus tendencias a 
lo largo del tiempo, que también se corroboran en los resultados presentados antes. Esto plantea la necesidad de confirmar esta tendencia por un periodo más largo de tiempo, ya que en estudios anteriores estas tendencias se han comprobado incluso con diferencias de años (Hederich y Camargo, 1999) y en este período de los niños dichas tendencias podrían variar por sus cambios en la comprensión de estados intencionales en los demás (Symons et al., 2005; Dunn y Brophy, 2005).

Una inquietud adicional queda con respecto a las acciones que se plantearon como indicadores en la lectura conjunta: son acciones que al parecer se relacionan más con los actos de lectura que con el apego o la cercanía afectiva en la interacción entre el niño y el adulto (Sierra, 2004; Hederich, 2007). Por tanto, es necesario estudiar otros indicadores más ligados a la tradición del estudio del apego pero que puedan dar cuenta también de la relación entre los niños y los adultos en su interacción mediada por los libros (Bus, 2002).

\section{Bloque correlacional}

Para poder hacer válido el indicador de estilo cognitivo es preciso que haya una correlación negativa o nula (nunca positiva) entre los puntajes de las pruebas EFT y RFT. La correlación de Pearson es negativa y significativa entre EFT y RFT $(r=-0,89, p<0,01)$. La puntuación agrupada de estilo cognitivo se calculó mediante la diferencia entre el puntaje estándar de la prueba EFT y el puntaje estándar en la prueba RFT.

Tomando como criterio la media y una desviación estándar por encima o por debajo de esta, se establecieron los grupos de padres de acuerdo con su estilo cognitivo (tabla 3). Se observa que hay dos díadas con adultos muy sensibles, cuatro con sensibles, tres con independientes y tres con muy independientes; es decir, la mayoría de los sensibles tienden a acercarse al intermedio del rango de puntuaciones de estilo cognitivo y los independientes tienden a distribuirse por igual entre los que se acercan a las puntuaciones intermedias y los que se acercan al extremo.

Tabla 3. Distribución de díadas de acuerdo con el estilo cognitivo del adulto.

\begin{tabular}{|c|c|c|}
\hline $\begin{array}{c}\text { Rango de puntuación de } \\
\text { estilo cognitivo }\end{array}$ & Rótulo de estilo cognitivo asignado & $\mathrm{n}$ \\
\hline$\leq-1,79$ & Muy sensible & 2 \\
\hline$[-1,78: 0,00]$ & Sensible & 4 \\
\hline$[0,01: 1,79]$ & Independiente & 3 \\
\hline$>1,79$ & Muy independiente & 3 \\
\hline
\end{tabular}


Las correlaciones de Spearman fueron moderadas en dos casos: estilo cognitivo del adulto independiente del medio y "niño habla con el adulto sobre elementos gráficos en el libro" (Rho de Spearman $=0,307, p=0,04$ ), y estilo cognitivo del adulto en independencia del medio y "niño expresa concentración en la actividad" (Rho de Spearman $=0,418 p=0,01$ ).

Se reconocen posibles fallas en las escalas y por ello se realizó un análisis de correspondencias múltiples para examinar tendencias de agrupación de las variables estilo cognitivo y patrones de lectura conjunta ${ }^{7}$. Según este análisis, se obtuvieron patrones de diferencia entre las acciones en los dos primeros factores, con valores propios de 0,2329, y 0,2093, respectivamente. Estos dos factores se escogieron para el resto del análisis y se desecharon los demás factores resultantes de acuerdo con el criterio de caída máxima de la pendiente8 (tabla 4 y figura 3 ).

7 El análisis de correspondencias múltiples es un cálculo equivalente a un análisis factorial que se efectúa con valores de variables nominales u ordinales. Esta estadística opera teniendo en cuenta chi cuadrado, un cálculo que opera con datos de tablas de contingencia. Chi cuadrado opera con conjuntos de datos independientes y con sus cruces, con independencia de su disposición en filas o columnas en su representación. Esto hace que en el análisis de correspondencias múltiples se pueda operar con conjuntos de datos con independencia de si se trata de variables o de sujetos en una matriz de datos. El funcionamiento de este análisis se describe con detalle en Hederich y Camargo (2000).

8 El criterio de mayor caída de la pendiente establece que se escogen como factores de agrupación o punto de corte entre número de factores escogidos y no escogidos para la agrupación el punto en el cual se encuentra la mayor distancia numérica entre dos factores seguidos. Al expresarse gráficamente en un diagrama de barras, dicha diferencia es observable en una caída con una pendiente mucho mayor entre el porcentaje de explicación o de tendencia de agrupación que tienen dos factores contiguos. Para el caso de presente análisis, este punto de corte se dio entre el tercero y el cuarto factor, por lo cual resultaron tres factores o dimensiones. Sin embargo, el tercer factor agrupaba acciones en las cuales no se pudo identificar un patrón de polarización claramente definido y se trabajó únicamente con los dos primeros factores. 
Tabla 4. Resultados resumidos del análisis de correspondencias múltiples.

\begin{tabular}{|c|c|c|}
\hline Factor & \multicolumn{2}{|c|}{ Acciones agrupadas } \\
\hline \multirow{7}{*}{$\begin{array}{l}\text { PRIMERO: } \\
\text { inercia } \\
\text { explicada: } \\
23,29 \%\end{array}$} & $\begin{array}{c}\text { Eje negativo } \\
\text { características agrupadas: alto control de } \\
\text { la actividad por el adulto y focalización } \\
\text { en el libro }\end{array}$ & $\begin{array}{c}\text { Eje positivo } \\
\text { características agrupadas: bajo control de } \\
\text { la actividad por el adulto y focalización } \\
\text { en el otro }\end{array}$ \\
\hline & $\begin{array}{l}\text { El adulto sí escoge el libro para el niño. } U \\
=-2,5\end{array}$ & $\begin{array}{l}\text { El adulto no escoge el libro para el niño. } \\
U=2,5\end{array}$ \\
\hline & $\begin{array}{l}\text { El niño sí habla con el adulto sobre ele- } \\
\text { mentos gráficos en el libro. } U=-2,2\end{array}$ & $\begin{array}{l}\text { El niño no habla con el adulto sobre ele- } \\
\text { mentos gráficos en el libro. } U=2,2\end{array}$ \\
\hline & $\begin{array}{l}\text { El niño no comenta todo el tiempo el } \\
\text { contenido de los libros. } U=-2,2\end{array}$ & $\begin{array}{l}\text { El niño sí comenta todo el tiempo el } \\
\text { contenido de los libros. } U=2,2\end{array}$ \\
\hline & $\begin{array}{l}\text { El niño se aparta todo el tiempo del adulto } \\
\text { y observa el libro solo. } U=-2,6\end{array}$ & $\begin{array}{l}\text { El niño no se aparta todo el tiempo del } \\
\text { adulto ni observa el libro solo. } U=2,6\end{array}$ \\
\hline & $\begin{array}{l}\text { El niño manifiesta interés por la lectura con- } \\
\text { junta solo hasta cierto momento. } U=-3,0\end{array}$ & $\begin{array}{l}\text { El niño manifiesta interés por la lectura } \\
\text { conjunta todo el tiempo } U=3,0\end{array}$ \\
\hline & $\begin{array}{l}\text { Ilustrativas: estilo cognitivo muy indepen- } \\
\text { diente }(U=-0,7) \text {, estilo cognitivo indepen- } \\
\text { diente }(U=-0,5) \text {. }\end{array}$ & $\begin{array}{l}\text { Ilustrativas: estilo cognitivo muy sensible } \\
(U=0,7) \text {, estilo cognitivo sensible }(U= \\
0,5) \text {, el niño disfruta yendo a la biblioteca } \\
(U=2,1) \text {, Si hay otro que lea en casa con } \\
\text { el niño }(U=1,6) \text {, el padre lee con el niño } \\
(U=1,6) \text {, Biblioteca Sistema Biblored ( } U \\
=2,0) \text {, Biblioteca Caja de Compensación } \\
(U=1,0)\end{array}$ \\
\hline \multirow{7}{*}{$\begin{array}{l}\text { SEGUNDO: } \\
\text { inercia } \\
\text { explicada: } \\
20,93 \%\end{array}$} & $\begin{array}{c}\text { Eje negativo } \\
\text { Preferencia por los aspectos narrativos del } \\
\text { texto }\end{array}$ & $\begin{array}{l}\text { Eje positivo } \\
\text { Preferencia por los aspectos gráficos y } \\
\text { temáticos del texto. }\end{array}$ \\
\hline & $\begin{array}{l}\text { El niño no habla con el adulto del tema y } \\
\text { de las imágenes del libro. } U=-2,5\end{array}$ & $\begin{array}{l}\text { El niño sí habla con el adulto del tema y } \\
\text { de las imágenes del libro. } U=2,5\end{array}$ \\
\hline & $\begin{array}{l}\text { El niño sí habla con el adulto todo el tiem- } \\
\text { po sobre situaciones específicas narradas. } \\
U=-2,6\end{array}$ & $\begin{array}{l}\text { El niño no habla con el adulto todo el } \\
\text { tiempo sobre situaciones específicas narra- } \\
\text { das. } U=2,6\end{array}$ \\
\hline & $\begin{array}{l}\text { El niño sí muestra todo el tiempo el libro a } \\
\text { los adultos acompañantes. } U=-2,2\end{array}$ & $\begin{array}{l}\text { El niño no muestra todo el tiempo el libro } \\
\text { a los adultos acompañantes. } U=2,2\end{array}$ \\
\hline & $\begin{array}{l}\text { El niño sí expresa alegría y entusiasmo } \\
\text { durante la actividad. } U=-1,9\end{array}$ & $\begin{array}{l}\text { El niño no expresa alegría y entusiasmo } \\
\text { durante la actividad. } U=1,9\end{array}$ \\
\hline & $\begin{array}{l}\text { El niño no expresa concentración durante } \\
\text { la actividad. } U=-1,6\end{array}$ & $\begin{array}{l}\text { El niño sí expresa concentración durante } \\
\text { la actividad. } U=1,6\end{array}$ \\
\hline & $\begin{array}{l}\text { llustrativas: madre con estudios superiores } \\
\text { incompletos }(U=-3,1) \text {, padre con estudios } \\
\text { superiores incompletos }(U=-2,0) \text {, cuidador } \\
\text { con bachillerato completo }(U=-2,0) \text {, } \\
\text { madre pequeña comerciante }(U=-2,1) \text {, } \\
\text { padre empleado del comercio }(U=-2,0) \text {, } \\
\text { madre de } 41 \text { a } 50 \text { años }(U=-2,1) \text {, padre de } \\
51 \text { años o más }(U=-2,1) \text {, órdenes flexibles } \\
\text { principio educativo más apreciado }(U= \\
-2,1) \text {, pocas costumbres fijas }(U=-2,0) \text {. }\end{array}$ & Sin ilustrativas asociadas \\
\hline
\end{tabular}

Nota: se consideran estadísticamente significativos los valores mayores a 1,9 y menores de -1,9. En esta tabla se incluyen otros con valores que no alcanzan a ser significativos pero que marcan de todas maneras una tendencia importante en los ejes descritos en el análisis de correspondencias múltiples. 
En el análisis de correspondencias múltiples se hallan dos tendencias o polaridades de agrupación de las acciones en la lectura conjunta: 1.más control yfocalización en el libro versus menos control con focalización en el otro y 2. preferencia por objetos gráficos y temáticos del texto versus preferencia por detalles narrativos del texto.

En la primera polaridad hay una oposición entre más control con focalización en el libro y menos control con focalización en el otro. El mayor control con focalización en el libro agrupa acciones como: la escogencia del libro para el niño por el adulto, el niño habla con el adulto sobre elementos gráficos en el libro, el niño se aparta todo el tiempo del adulto y observa el libro solo y el niño manifiesta interés por la lectura solo hasta cierto momento. Adicionalmente, tiene tendencia a asociarse con el estilo cognitivo independiente y muy independiente del medio en el adulto. El menor control con focalización en el otro agrupa acciones como: el niño comenta todo el tiempo el contenido de los libros y el niño manifiesta interés por la lectura conjunta todo el tiempo. También tiende a asociarse fuertemente con el niño disfruta asistir a la biblioteca, hay otro que lee en casa con el niño, el padre lee con el niño y uso de sistemas de bibliotecas públicas y de cajas de compensación, y a asociarse débilmente con estilo cognitivo sensible y muy sensible al medio en el adulto.

La segunda polaridad muestra una oposición entre el privilegio a aspectos narrativos y gráficos del texto, además de la temática general del texto. El privilegio a aspectos narrativos en el texto agrupa las acciones: el niño habla todo el tiempo con el adulto sobre situaciones específicas narradas, el niño muestra todo el tiempo el libro a los adultos acompañantes y el niño expresa alegría y entusiasmo durante la actividad. Se asocian con estudios superiores incompletos en la madre y el padre, tener un cuidador con educación secundaria y media, independencia como cualidad más deseada, órdenes flexibles como el principio educativo más valorado, ocupaciones del comercio en los padres, edades superiores a 41 años en los padres y pocas costumbre fijas en la vida del hogar. El privilegio a aspectos gráficos y de temática general del texto agrupa las acciones: el niño habla con el adulto del tema y de las imágenes del libro, y el niño expresa concentración durante la actividad. 


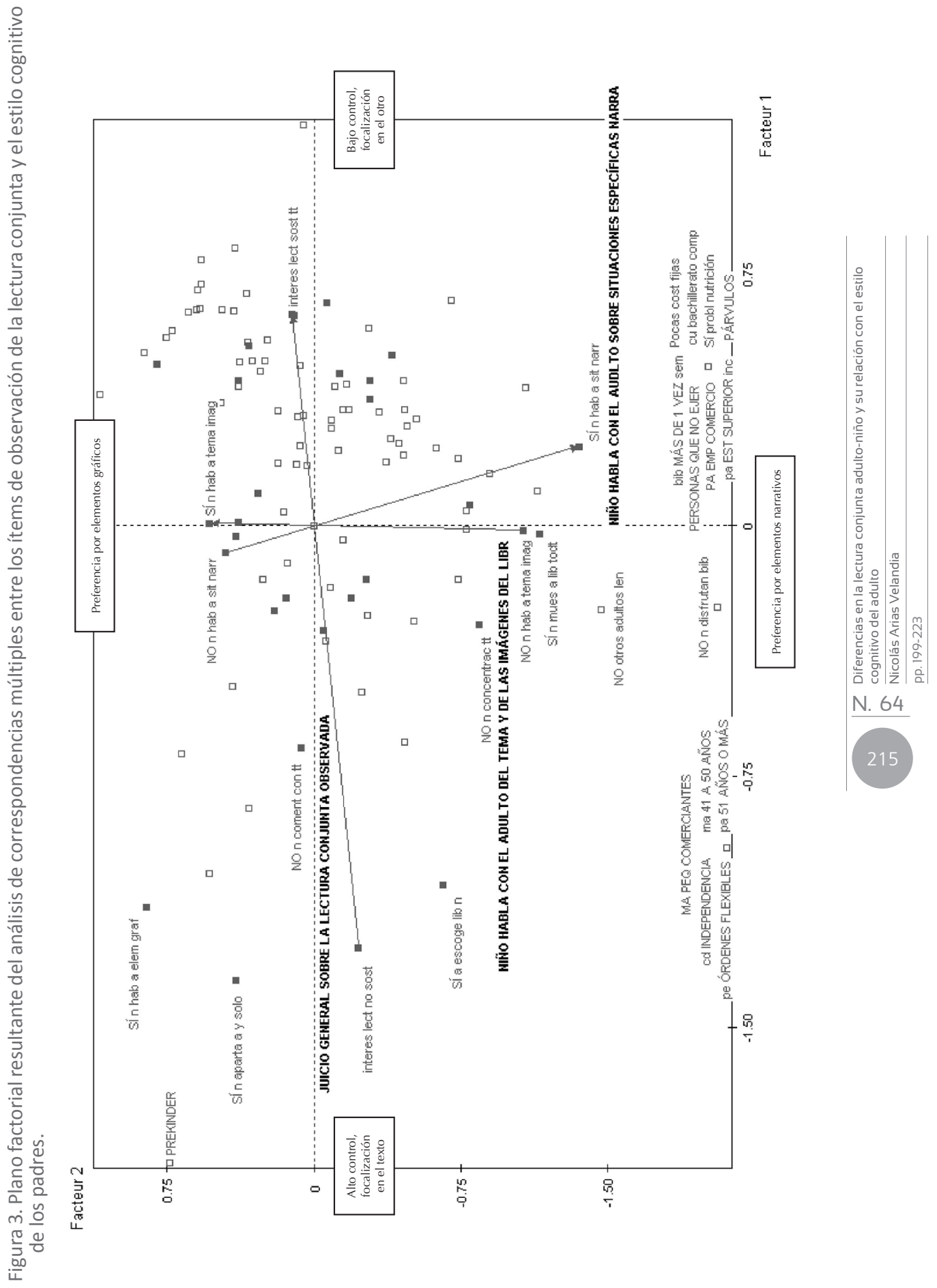




\section{Discusiones del bloque correlacional}

Una de las dimensiones de agrupación de las acciones en la lectura conjunta es la de control y foco de la actividad. En esta se encontraron dos polos de agrupación: alto control del adulto con focalización en el libro y bajo control del adulto con focalización en el otro. Este hallazgo coincide con el planteamiento de Bus (2002), en el cual se clasifica las tendencias de interacción en díadas en la lectura conjunta entre los que se centran en el objeto de la actividad (el libro) y los que lo hacen en la interacción misma y en la reacción con el otro a cada momento.

Adicionalmente, también se encuentran asociados en estos resultados en una dimensión de mayor o menor control de la interacción. Queda la duda de si esto es algo propio de la edad de los niños y no necesariamente una tendencia de estilo: Bus (2002) halla que estas tendencias pueden variar con la edad del niño y con las expectativas de desempeño que de él tienen los padres y adultos cercanos. Como este trabajo se realizó con pequeños de un solo grupo de edad, esta duda es válida a pesar nuestra evidencia de que se puede tratar de una dimensión de estilo (Hederich, 2007).

También se halló una relación importante, aunque débil, entre el estilo cognitivo del adulto y la dimensión de control y foco de la actividad encontrada en la lectura conjunta. La presencia de esta relación, sumada a que parece mostrar también consistencia temporal, consistencia entre diferentes tipos de acciones y poca relación con variables como el nivel educativo, ocupación y valoraciones de los padres, hace pensar razonablemente en que al menos esta dimensión de la lectura conjunta sería una dimensión con características de estilo (Saracho, 2003; Hederich, 2007). Esto coincide con una de las principales hipótesis de los estudiosos del estilo cognitivo en la dimensión independencia - sensibilidad al medio acerca de la tendencia integradora de dimensiones de la actividad que tienen los estilos en la vida del ser humano, y esta podría ser una de sus manifestaciones (Hederich, 2007).

Sin embargo, la relación con el estilo cognitivo es débil, lo cual hace necesario retomar estos estudios con una muestra más grande de personas y con indicadores más precisos, con el fin de establecer con más firmeza si efectivamente hay una relación entre las dimensiones en la lectura conjunta y el estilo cognitivo de los adultos que guían la actividad. 
Los resultados también arrojan otra dimensión: la preferencia del niño por elementos significativos del texto, que también es reseñada por Bus (2002). Como lo indican las relaciones con otros factores, esta dimensión se relaciona con características demográficas del adulto con quien se realiza la actividad y con características de cercanía a actividades propias de familiaridad con el mundo de la lectura. Esto tiene dos posibles interpretaciones: o bien se trata de una diferencia de nivel (y no de estilo) que refleja factores de un medio alfabético enriquecido (Bus y Sulzby, 1996; Evans et al., 2000; Van Kleeck, 2003; Collins et al., 2005; Flórez et al., 2006a, 2006b; Flórez et al., 2007), o se trata de la adopción de un modo de conversación en los padres que es propio de algunos grupos culturales (Lee et al., 2004). Un conjunto de evidencias más grande nos podría iluminar si es válida la primera o segunda opción de interpretación.

Otro hallazgo que muestra este estudio es que las acciones en la lectura conjunta tienen distintas formas de agrupación de acuerdo con el énfasis que marcan en la forma de hablar y dirigir la conversación hacia propósitos específicos en esta actividad (Jensen y Kolb, 2000; Saracho, 2000, 2003). En ese sentido, muestra diferentes dimensiones como polaridades que marcan diferencias muy importantes en estos puntos, lo cual indica que, en principio, estas polaridades pueden ser también indicadores de un estilo de comunicación en la interacción dirigida a propósitos de aprendizaje (Camargo y Hederich, 2007).

\section{Conclusiones y recomendaciones}

Este estudio muestra que existe un conjunto de acciones que se presenta en todas las díadas, relacionadas con el mantenimiento de la interacción. Del mismo modo, existe un conjunto de acciones en las que varía su aparición en las díadas: en la tendencia fuerte se polarizan entre los que privilegian entre el contenido y la interacción, y en una débil los que polarizan entre lo narrativo y lo gráfico.

Otros factores relacionados con los reportes de lectura y aprendizaje en el hogar muestran una relación importante únicamente con el privilegio de lo narrativo en la interacción del niño y el adulto con el texto. Por otra parte, el estilo cognitivo del adulto solamente muestra una relación débil con la polaridad de control y focalización de la interacción durante la lectura conjunta, con una tendencia de los adultos independientes del medio a dirigir interacciones con mayor control y focalización en el texto, y con una tendencia de los adultos sensibles al medio a mantener interacciones con menor control y focalización en el niño.

Estas tendencias son interesantes pero no concluyentes, por lo cual se hace necesario continuar este estudio con una muestra más extensa 
de diadas participantes, y precisar las definiciones de algunas acciones y complementarlas con otras que amplíen el panorama de observación de las situaciones de lectura conjunta. Del mismo modo, es necesario asegurar la fuerza de estos resultados a través de más observaciones de las interacciones a través del tiempo y ver su relación con pruebas de progresos en el aprendizaje de la lectura (o de sus inicios) en niños pequeños.

Este estudio muestra también, de manera indirecta, la importancia de implementar estrategias de apoyo a la lectura y la escritura inicial, de realizar acciones de enseñanza que resalten el libro como objeto y la interacción alrededor de contactos con él (así como la de realizar acciones que resalten los aspectos gráficos y narrativos en relación con el libro). Es posible entonces que las díadas con una determinada tendencia de interacción o estilo cognitivo en el adulto adquieran recursos adicionales para su actividad de lectura conjunta con el niño que les den un conjunto adicional de recursos que potencie dicha actividad y sus efectos en el aprendizaje inicial de la lectura en el niño (cf., Hederich, 2007; Saracho, 2003).

\section{Agradecimientos}

El autor agradece al profesor Christian Hederich por la asesoría a la tesis que soporta este artículo. Agradecemos también a Lucila Obando Velásquez y Geral Eduardo Mateus Ferro, profesores del Departamento de Lenguas de la Universidad Pedagógica Nacional, por su aporte a este trabajo con sus anotaciones críticas como jurados del trabajo de grado que da origen a este artículo. Se reconoce también el trabajo de lectura de la profesora Ángela Camargo Uribe, del mismo departamento, y el apoyo logístico y en tiempo de las profesoras Rita Flórez Romero y Yolanda Rueda Delgado, en la Universidad Nacional de Colombia. Reconocemos también el apoyo institucional de la Maestría en Educación, el Departamento de Posgrados de la Facultad de Educación y el Centro de Investigaciones CIUP, todos de la Universidad Pedagógica Nacional, y de la Universidad Nacional de Colombia. También agradecemos a los niños y padres de familia participantes por su participación y por haber hecho posible este estudio. 


\section{Referencias}

Bus, A. (2002). Joint Caregiver Child Storybook Reading: A Route to Literacy Development. En S. B. Neuman y D. K. Dickinson (eds.) Handbook of Early Literacy Research (pp. 179-191). Nueva York: The Guilford Press.

Bus, A. y Sulzby, E. (1996). Becoming Literate in a Multicultural Society. En J. Shimron (ed.). Literacy and Education: Essays in memory of Dina Feitelson (pp. 31-45). Cresskill, NJ: Hampton Press.

Camargo, A., y Hederich, C. (2007). El estilo de comunicación y su presencia en el aula de clase. Folios, 26, 3-12.

Collins, F.; Svensson, C. y Mahony, P. (2005). Bookstart: Planting a Seed for Life. Final Report. Londres: Roehampton University.

Cuervo, C.; Flórez, R. y Acero, G.A. (2004). El desarrollo del lenguaje: de lo oral a lo escrito. En R. Flórez (ed.). El lenguaje en la educación (pp. 27-84). Bogotá: Departamento de la Comunicación Humana y sus Desórdenes, Facultad de Medicina, Universidad Nacional de Colombia.

Cuetos, F. (2002). Psicología de la Lectura. 4a. ed. Barcelona: Praxis.

Dickinson, D.K.; McCabe, A.; Anastasoupuolos, L.; PeisnerFeinberg, E. y Poe, M. (2003). The comprehensive language approach to early literacy: the interrelationships among vocabulary, phonological sensitivity and print knowledge among preschool-aged children. Journal of Educational Psychology 95 (3), 465-481.

Dunn, J. y Brophy, M. (2005). Communication, relationships, and individual differences in children $s$ understanding of mind. En J.W. Astington y J. Baird (eds.). Why Language Matters For Theory Of Mind (pp. 5069). London: Oxford University Press.

Evans, M.A.; Shaw, D. y Bell, M. (2000). Home literacy activities and their influence on early literacy skills. Canadian Journal of Experimental Psychology 54 (2), 65-75.

Flórez, R.; Castro, J.A. y Arias, N. (2009). Comunicación, lenguaje y educación: una mirada desde las teorías de la complejidad. Folios, 30, 25-38.

Flórez, R.; Restrepo, M. A. y Schwanenflugel, P. (2007). Alfabetismo Emergente: Investigación, Teoría y Práctica. El caso de la lectura. Bogotá: Departamento de la Comunicación Humana y Sus Desórdenes, Universidad Nacional de Colombia / Instituto para la Investigación Educativa y el Desarrollo Pedagógico, Secretaría de Educación Distrital de Bogotá, D. C.

Flórez, R.; Torrado, M.C. y Arias, N. (2006a). Leer en familia: horizonte conceptual. En Fun- 
dalectura (ed.). Leer en familia en Colombia. Reporte de investigación y experiencias (pp. 19-45). Bogotá: Editor.

Flórez, R.; Torrado, M.C. y Arias, N. (2006b). Los bebés también pueden leer en familia: resultados de una experiencia en Bogotá. En Fundalectura (ed.). Leer en familia en Colombia. Reporte de investigación y experiencias (pp. 49-62). Bogotá: Editor.

Hederich, C. (2007). Estilo cognitivo en la dimensión de dependencia-independencia de campo. Influencias culturales e implicaciones para la educación. Colección Tesis Doctorales. Bogotá: Universidad Pedagógica Nacional.

Hederich, C. y Camargo, A. (1999). Estilos cognitivos en Colombia. Bogotá: Universidad Pedagógica Nacional-Colciencias.

Hederich, C. y Camargo, A. (2000). Estilo cognitivo y logro en el sistema educativo de la ciudad de Bogotá. Bogotá: Universidad Pedagógica Nacional, Instituto para la Investigación Educativa y el Desarrollo Pedagógico -IDEP-

Jensen, P.J. y Kolb, D.A. (2000). Learning style and meaning making in conversation. En R. J. Riding y S.G. Rayner (eds.). International Perspectives on Individual Differences (pp. 277299). Vol. 1, Cognitive Styles. Stanford, Conn: Ablex.

Lautrey, J. (1985). Clase social, medio familiar e inteligencia. Madrid: Aprendizaje Visor.

Lee, C.; Daniel, K. y Langellier, M. (2004). Cultural Modeling as a framework for Narrative Analysis. En C. Daiute y C. Lightfoot (eds.). Narrative Analysis: Studying the Development of Individuals in Society (pp. 39-61). Londres: Sage.

Leseman, P.M. y De Jong, P.F. (1998). Home literacy: Opportunity, instruction, cooperation social-emotional quality predicting early reading achievement. Reading Research Quarterly, 33, 294-318.

Páramo, M.F.; Dosil, A. y Tinajero, C. (1991). An Approach to Family Antecedents of Field Dependent - Independent Cognitive Style Based on Lautrey's Model. Cahiers de Psychologie Cognitive - European Bulletin of Cognitive Psychology 11 (5), 605-618.

Páramo, M.F. y Tinajero, C. (1990). Interacción familiar y estilo cognitivo. Bases para una posible intervención. Cuadernos de Terapia Familiar, 14, IV, 25-33.

Páramo, M.F. y Tinajero, C. (1992). Influencia de la estructura normativa familiar sobre el estilo cognitivo dependencia - 
independencia de campo: un estudio prospectivo. Infancia y Aprendizaje, 57, 89-98.

Perinat, A. (1999). Psicología del desarrollo. Un enfoque sistémico. Barcelona: EDHUIOC.

Saracho, O. (2000). A framework for effective classroom teaching: matching teachers' and students' cognitive styles. En R.J. Riding y S.G. Rayner (eds.). International Perspectives on Individual Differences (pp. 297314). Vol. 1, Cognitive Styles. Stanford, Conn: Ablex.

Saracho, O. (2003). Matching Teachers' and Students' Cognitive Styles. Early Child Development and Care 173 (2-3), 161-173.

Sénéchal, M.; LeFebvre, J.A.; Hudson, E. y Lawson, E.P. (1996). Knowledge of storybooks as a predictor of young children's vocabulary. Journal of Educational Psychology, 88, 520-536.

Sierra, H. (2004). La emoción como proceso cognitivo. Diálogos, Discusiones en la Psicología Contemporánea, 3, 123-142.

Storch, R. y Whitehurst, G.J. (2002). Oral language and code-related precursors to reading. Evidence from longitudinal structural model. Developmental Psychology 38 (6), 934-947.

Symons, D.K.; Peterson, C.C.; Slaughter, V.; Roche, J. y Doyle, E. (2005). Theory of mind and mental state discourse during book reading and story telling tasks. British Journal of Developmental Psychology, 23, 81-102.

Van Kleeck, A. (2003). Research on Book Sharing: Another Critical Look. En A. Van Kleeck, S. Stahl y E. Bauer (eds.). On Reading Book to Children: Parents and Teachers. Mawah, NJ: Lawrence Erlbaum Associates.

Wade, B. y Moore, M. (2000). A sure start with books. Early Years 20 (2), 39-46.

Watson, R. (2002). Literacy and oral language: implication for early literacy acquisition. En S.B. Neuman y D.K. Dickinson (eds.). Handbook of Early Literacy Research (pp. 43-53). Nueva York: The Guilford Press.

Wells, G. (1981). Learning Through Interaction. Cambridge: Cambridge University Press.

Wells, G. (1986). The Meaning Makers. Londres: Hodder \& Stoughton.

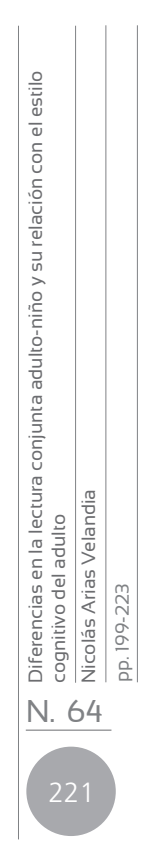




\section{Anexo 1. Guía de observación de la actividad de lectura}

fecha de esta observación:

Nombre del niño:

Fecha de nacimiento: Edad:

Observador: Biblioteca ¿Quién hace la lectura con el niño?: Mamá ___ Papá ___ Otro adulto ¿quién? Estado de salud del niño en el momento de la observación: Favorable Estado de ánimo del niño en el momento de la observación: Favorable Desfavorable Desfavorable

1) Comienzo de la actividad. Observe si se presentan los siguientes comportamientos en el momento de iniciar la lectura conjunta, y marque una equis $(\mathrm{X})$ en la casilla correspondiente al frente de lo observado:

A. Al iniciar la actividad el niño se emociona o muestra una reacción particular cuando se le invita a leer

\begin{tabular}{|l|l|l|}
\hline \multirow{2}{*}{ B. El adulto } & a. Escoge un libro para el niño. & \\
\cline { 2 - 3 } & b. permite o invita al niño a que él mismo escoja el libro. & \\
\hline
\end{tabular}

2) Curso de la actividad. Observe si se presentan los siguientes comportamientos durante la lectura conjunta, $y$ marque una equis $(X)$ en la casilla correspondiente:

\begin{tabular}{|l|l|}
\hline A. El adulto: & \\
\hline a. lee de corrido con el niño sin atender mucho a sus comentarios & \\
\hline b. lee con el niño y conversa activamente con él de lo que leen & \\
\hline c. deja que el niño juegue solo con los libros & \\
\hline
\end{tabular}

B. El adulto modifica su tono de voz al leer con el niño

\begin{tabular}{|l|l|}
\hline C. El adulto lee o muestra: & \\
\hline a. todo el contenido del libro al niño & \\
\hline b. solo una parte del contenido del libro al niño & \\
\hline
\end{tabular}

D. El niño:

a. se siente tenso, incómodo o intenta desasirse del adulto para eludir la actividad

b. toma el libro en sus manos y continúa solo la actividad

c. participa activamente en la actividad.

E. El niño, cuando intenta seguir la lectura solamente:

a. se lleva el libro en las manos solamente.

b. parece reproducir o volver a contar lo que el adulto les ha leído antes.

c. le muestra el libro al adulto o le dice algo sobre él.

\begin{tabular}{|l|l|l|}
\hline \multirow{3}{*}{$\begin{array}{l}\text { F. Durante la lectura, el } \\
\text { niño habla con el adulto }\end{array}$} & a. la parte escrita del libro. & \\
\cline { 2 - 3 } acerca de: & b. el tema y las imágenes del libro. & \\
\cline { 2 - 3 } & c. otros elementos gráficos en el libro. & \\
\cline { 2 - 3 } & d. situaciones específicas contadas en los libros. & \\
\hline
\end{tabular}

\begin{tabular}{|l|l|}
\hline G. El niño todo el tiempo: & \\
\hline a. muestra el libro a los adultos que le acompañan & \\
\hline b. explora diferentes libros & \\
\hline c. comenta el contenido de los libros & \\
\hline d. se aparta de los padres y observa el libro a solas & \\
\hline
\end{tabular}


3) Finalización de la actividad. Observe si se presentan los siguientes comportamientos al terminar la lectura conjunta:

A. El niño pide continuar cuando el adulto lo invita a dejar la lectura

\begin{tabular}{|l|l|}
\hline B. Al terminar la sesión de lectura, el niño: & \\
\hline a. Agarra el libro sin soltarlo. & \\
\hline b. Agarra y mira el libro sin el adulto cuidador. & \\
\hline c. Toma otro libro y pide al adulto que se lo lea. & \\
\hline
\end{tabular}

\begin{tabular}{|l|l|}
\hline \multicolumn{2}{|l|}{ C. Además del adulto que guía al niño, se ha involucrado otra persona en la lectura } \\
\hline a. Otro adulto & \\
\hline b. Otro niño & \\
\hline c. El promotor & \\
\hline
\end{tabular}

D. ¿Algún participante o adulto familiar presta libros a domicilio en la actividad?

4) Observaciones posteriores a la actividad. Indique si se presentan los siguientes comportamientos durante la lectura conjunta:

\begin{tabular}{|l|l|}
\hline A. El niño expresa alguna o varias de las siguientes reacciones emocionales durante la actividad: \\
\hline a. alegría y entusiasmo & \\
\hline c. concentración & \\
\hline
\end{tabular}

B. El adulto en la actividad, casi todo el tiempo prefiere:

a. hablar sobre los dibujos con el niño

d. dejar que el niño sostenga y manipule el libro mientras el mismo guía la lectura

e. dejar que el niño guíe toda la actividad

5) Marque con una equis $(X)$ el criterio que corresponda a la observación realizada:

\begin{tabular}{|l|l|}
\hline A. Cuando el adulto lee en voz alta: \\
\hline a. el niño se interesa por la lectura todo el tiempo & \\
\hline b. el niño se interesa por la lectura solo hasta cierto momento & \\
\hline c. el niño se interesa pero pierde rápidamente el interés & \\
\hline $\begin{array}{l}\text { d. el niño se concentra en la actividad por algunos momentos y vuelve a desconcentrarse } \\
\text { rápidamente }\end{array}$ & \\
\hline e. el niño muestra franco desagrado por la actividad o no muestra mayor interés en ella & \\
\hline
\end{tabular}

Escriba algunas observaciones particulares sobre algunos aspectos de la actividad que hayan llamado su atención: 